\title{
The effect of irregular breathing patterns on internal target volumes in four-dimensional CT and cone-beam CT images in the context of stereotactic lung radiotherapy
}

Clements, Natalie; Kron, Tomas; Franich, Rick; Dunn, Leon; Roxby, P; Aarons, Y; Chesson, B https://researchrepository.rmit.edu.au/esploro/outputs/9921858517501341/filesAndLinks?institution=61RMIT_INST\&index=null

Clements, N., Kron, T., Franich, R., Dunn, L., Roxby, P., Aarons, Y., Chesson, B., Siva, S., Duplan, D., \& Ball, D. (2013). The effect of irregular breathing patterns on internal target volumes in four-dimensional CT and cone-beam CT images in the context of stereotactic lung radiotherapy. Medical Physics - Radiation Imaging Physics, 40(2), 021904-1-021904-021910. https://doi.org/10.1118/1.4773310 Document Version: Accepted Manuscript

Published Version: https://doi.org/10.1118/1.4773310 
Thank you for downloading this document from the RMIT Research Repository.

The RMIT Research Repository is an open access database showcasing the research outputs of RMIT University researchers.

RMIT Research Repository: http://researchbank.rmit.edu.au/

\section{Citation:}

Clements, N, Kron, T, Franich, R, Dunn, L, Roxby, P, Aarons, Y, Chesson, B, Siva, S, Duplan, D and Ball, D 2013, 'The effect of irregular breathing patterns on internal target volumes in four-dimensional CT and cone-beam CT images in the context of stereotactic lung radiotherapy', Medical physics - Radiation Imaging Physics, vol. 40, no. 2, pp. 021904-1-021904-10

See this record in the RMIT Research Repository at: http://researchbank.rmit.edu.au/view/rmit:20434

Version: Accepted Manuscript

Copyright Statement: (c) 2013 Am. Assoc. Phys. Med. 0

Link to Published Version:

http://dx.doi.org/10.1118/1.4773310 


\title{
The Effect of Irregular Breathing Patterns on Internal Target Volumes in Four-Dimensional CT and Cone-Beam CT Images in the Context of Stereotactic Lung Radiotherapy
}

\author{
Clements, N. ${ }^{(a), 1,2}$, Kron, T. ${ }^{1}$, Franich, R. ${ }^{2}$, Dunn, L. ${ }^{2}$, Roxby, P. ${ }^{1}$, \\ Aarons, Y. ${ }^{3}$, Chesson, B. ${ }^{3}$, Siva, S. ${ }^{4}$, Duplan, D. ${ }^{4}$, Ball, D. ${ }^{4}$ \\ ${ }^{1}$ Department of Physical Sciences, Peter MacCallum Cancer Centre, East \\ Melbourne, Australia \\ ${ }^{2}$ Department of Applied Sciences, RMIT University, Melbourne, Australia \\ ${ }^{3}$ Department of Radiation Therapy, Peter MacCallum Cancer Centre, East \\ Melbourne, Australia \\ ${ }^{4}$ Department of Radiation Oncology, Peter MacCallum Cancer Centre, East \\ Melbourne, Australia
}

\section{ABSTRACT}

Purpose: Stereotactic lung radiotherapy is complicated by tumour motion from patient respiration. Four-dimensional CT (4DCT) imaging is a motion compensation method used in treatment planning to generate a maximum intensity projection (MIP) internal target volume (ITV). Image guided radiotherapy during treatment may involve acquiring a volumetric cone-beam CT (CBCT) image and visually aligning the tumour to the planning 4DCT MIP ITV contour. Moving targets imaged with CBCT can appear blurred and currently there are no studies reporting on the effect that irregular breathing patterns have on CBCT volumes and their alignment to 4DCT MIP ITV contours. The objective of this work was therefore to image a phantom moving with irregular breathing patterns to determine whether any configurations resulted in errors in volume contouring or alignment.

Methods: A Perspex thorax phantom was used to simulate a patient. Three wooden 'lung' inserts with embedded Perspex 'lesions' were moved up to $4 \mathrm{~cm}$ with computer-generated motion patterns, and up to $1 \mathrm{~cm}$ with patient-specific breathing patterns. The phantom was imaged on 4DCT and CBCT with the same acquisition settings used for stereotactic lung patients in the clinic and the volumes on all 
phantom images were contoured. This project assessed the volumes for qualitative and quantitative changes including volume, length of the volume, and errors in alignment between CBCT volumes and 4DCT MIP ITV contours.

Results: When motion was introduced 4DCT and CBCT volumes were reduced by up to $20 \%$ and $30 \%$ and shortened by up to $7 \mathrm{~mm}$ and $11 \mathrm{~mm}$, respectively, indicating that volume was being underrepresented at the extremes of motion. Banding artefacts were present in 4DCT MIP images, while CBCT volumes were largely reduced in contrast. When variable amplitudes from patient traces were used and CBCT ITVs were compared to 4DCT MIP ITVs there was a distinct trend in reduced ITV with increasing amplitude that was not seen when compared to true ITVs. Breathing patterns with a rest period following expiration resulted in well-defined superior edges and were better aligned using an edge-to-edge alignment technique. In most cases sinusoidal motion patterns resulted in the closest agreements to true values and the smallest misalignments.

Conclusions: Strategies are needed to compensate for volume losses at the extremes of motion for both 4DCT MIP and CBCT images for larger and varied amplitudes, and for patterns with rest periods following expiration. Lesions moving greater than 2 $\mathrm{cm}$ would warrant larger treatment margins added to the 4DCT MIP ITV to account for the volume being underrepresented at the extremes of motion. Lesions moving with a rest period following expiration would be better aligned using an edge-to-edge alignment technique. Sinusoidal patterns represented the ideal clinical scenario, reinforcing the importance of investigating clinically relevant motions and their effects on 4DCT MIP and CBCT volumes. Since most patients do not breathe sinusoidally this may lead to misinterpretation of previous studies using only sinusoidal motion.

Key words: stereotactic lung radiotherapy, irregular breathing pattern, 4DCT ITV, CBCT, alignment

a) Author to whom correspondence should be addressed. Electronic mail: natalie.clements@petermac.org; Telephone: +61 39928 8963; Fax: +61 396504870. 


\section{INTRODUCTION}

The complexity of stereotactic radiotherapy, typified by very large radiation doses in a few fractions, is further complicated by motion of the lung tumour caused by patient respiration. Four-dimensional CT (4DCT) imaging is commonly used to generate an internal target volume (ITV) contour that represents the volume in which the tumour moved throughout patient respiration. This ITV contour, from a maximum intensity projection (MIP) image, is then used to generate the radiotherapy plan. Any error in ITV contouring as a result of inaccurate imaging could result in a systematic error carried through to patient treatment. Cone-beam CT (CBCT) is a common volumetric imaging modality used to align the stereotactic lung patient prior to treatment by matching the visible tumour volume to the ITV contour on the planning CT scan. Cone-beam CT imaging is popular because the imager is conveniently mounted on the treatment machine; however, due to its slow ( $60 \mathrm{~s})$ imaging and blurred reconstructions of moving targets it may be unable to fully capture the tumour excursion

Patient alignment using 3D CBCT and planning scan contours is currently done with a best-fit centre-to-centre alignment of the visible CBCT volume to the planning CT scan. If blurring from motion reduced the visibility of the tumour on $\mathrm{CBCT}$ then this may affect centre-to-centre alignment (Fig. 1). Four-dimensional CT ITVs from sinusoidal and irregular breathing patterns have been analysed volumetrically and are reported in the literature, ${ }^{1-3}$ as have CBCT ITVs from sinusoidal patterns. ${ }^{2,4}$ However, the effect that irregular patterns have on CBCT ITVs has not yet been analysed in $3 \mathrm{D}$, only $2 \mathrm{D},{ }^{5}$ and there have been no reports on the alignment of CBCT ITVs to 4DCT MIP ITV contours as a result of irregular breathing patterns. This project addresses both of these previously unreported aspects.

The aim of this work is to investigate the effect that irregular breathing patterns have on the tumour ITV and alignment of the two imaging modalities used to plan and align the stereotactic lung patient; namely 4DCT maximum intensity projection (MIP) images and CBCT, respectively. The term ITV is used here to define the volumes in which the lesion moved on both 4DCT MIP and CBCT images. To accomplish the aim of this work, various lung lesions in a thorax phantom were moved with regular and irregular patterns of motion, imaged using 4DCT and CBCT, and all ITVs were analyzed both qualitatively and quantitatively. Clinical relevance 
was a focus of this work so the imaging acquisition settings and contouring tools used were the same as would be used for stereotactic lung patients in the clinic.

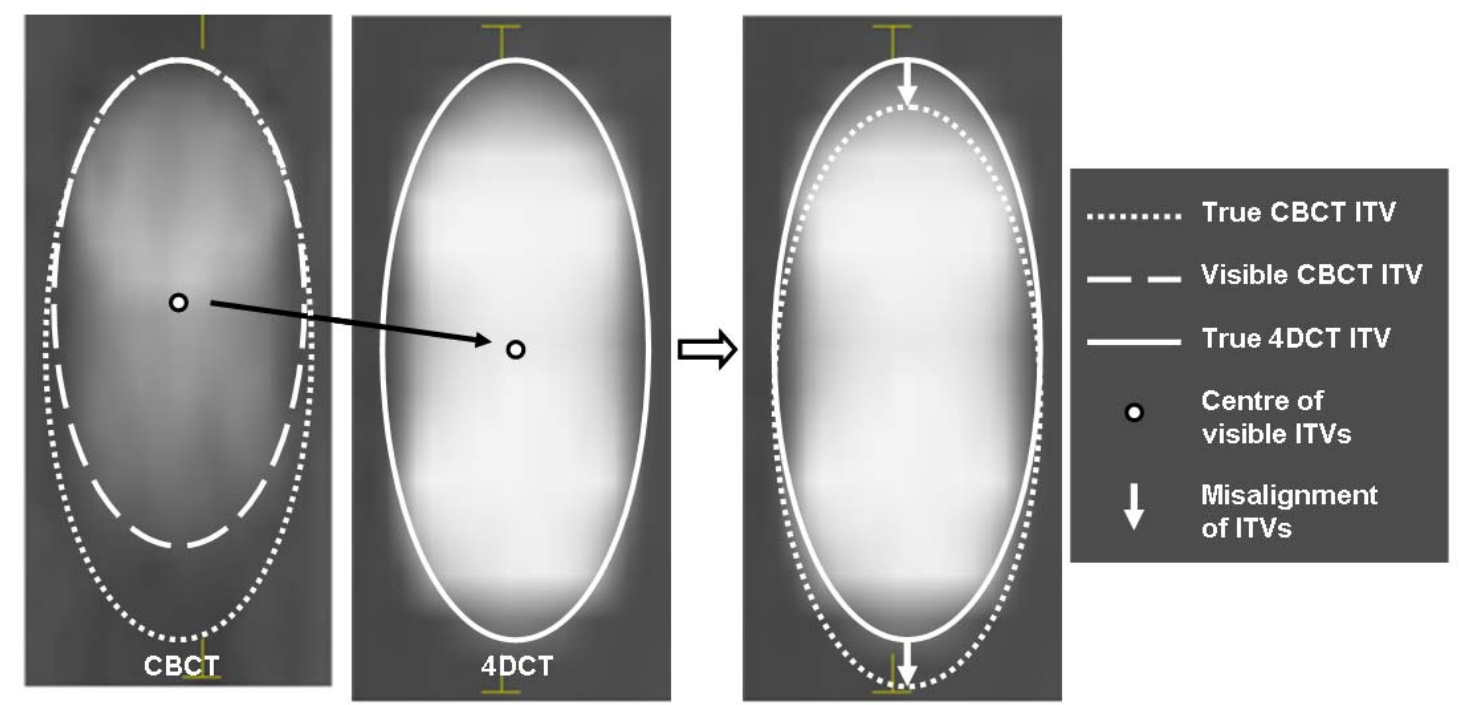

FIG. 1. The potential error in alignment from centre-to-centre ITV matching of a moving lesion moving. The visible CBCT ITV is smaller than the true CBCT ITV and may shift the ITV centre away from the centre of motion, resulting in a misalignment to the true 4DCT ITV contour.

\section{MATERIALS AND METHODS}

\section{II.A. Phantom equipment}

A QUASAR ${ }^{\mathrm{TM}}$ Programmable Respiratory Motion Phantom (Modus Medical Devices Inc., London, Ontario, Canada) was used to simulate a lung SBRT patient, driven using both generated and patient-specific breathing patterns.

A MATLAB script was used to import patient respiratory data and generate irregular motion patterns. These commands were uploaded to Modbus motor control software.

Three different sized Perspex 'lesions' centrally embedded in wooden 'lung' inserts were custom built for this project to reflect both the range of tumour volumes treated with lung SBRT and the imaging contrast between tumour and healthy lung tissue. The wooden cylinders $\left(\rho \sim 0.40 \mathrm{~g} / \mathrm{cm}^{3}\right)$ were $8 \mathrm{~cm} \mathrm{x} 17.5 \mathrm{~cm}$ (diameter $\mathrm{x}$ length), and the Perspex lesions $\left(\rho \sim 1.13 \mathrm{~g} / \mathrm{cm}^{3}\right)$ were cylindrical in shape with diameters and heights of $15 \times 15 \mathrm{~mm}, 30 \times 30 \mathrm{~mm}, 40 \times 40 \mathrm{~mm}$, and volumes of 2.65 
$\mathrm{cm}^{3}, 21.21 \mathrm{~cm}^{3}, 50.27 \mathrm{~cm}^{3}$, respectively. The phantom design constrained the lung insert motion along the superior-inferior (SI) axis.

Four computer-generated breathing patterns were created: a) sinusoidal, b) inspiration length less than expiration, c) inspiration length greater than expiration, and d) inspiration length roughly equal expiration followed by a rest period (Fig. 2). For these breathing patterns the lesions reached the peak amplitudes at each extreme.

Six SBRT lung patient breathing traces were also used to move the inserts in the QUASAR ${ }^{\mathrm{TM}}$ phantom. These patterns were captured using Varian ${ }^{\circledR}$ RPM system during $\mathrm{CBCT}$ acquisition at treatment mock-up for the patient and processed by the MATLAB-based stepper motor controller software.

The peak to peak amplitudes of motion used for the four computer-generated breathing patterns included $1 \mathrm{~cm}, 2 \mathrm{~cm}$, and $4 \mathrm{~cm}$ and these were adjusted manually on the phantom. The maximum amplitudes used for the six SBRT lung patient breathing patterns matched the patients' SI tumour excursions as seen on 4DCT, and are listed in Table III.
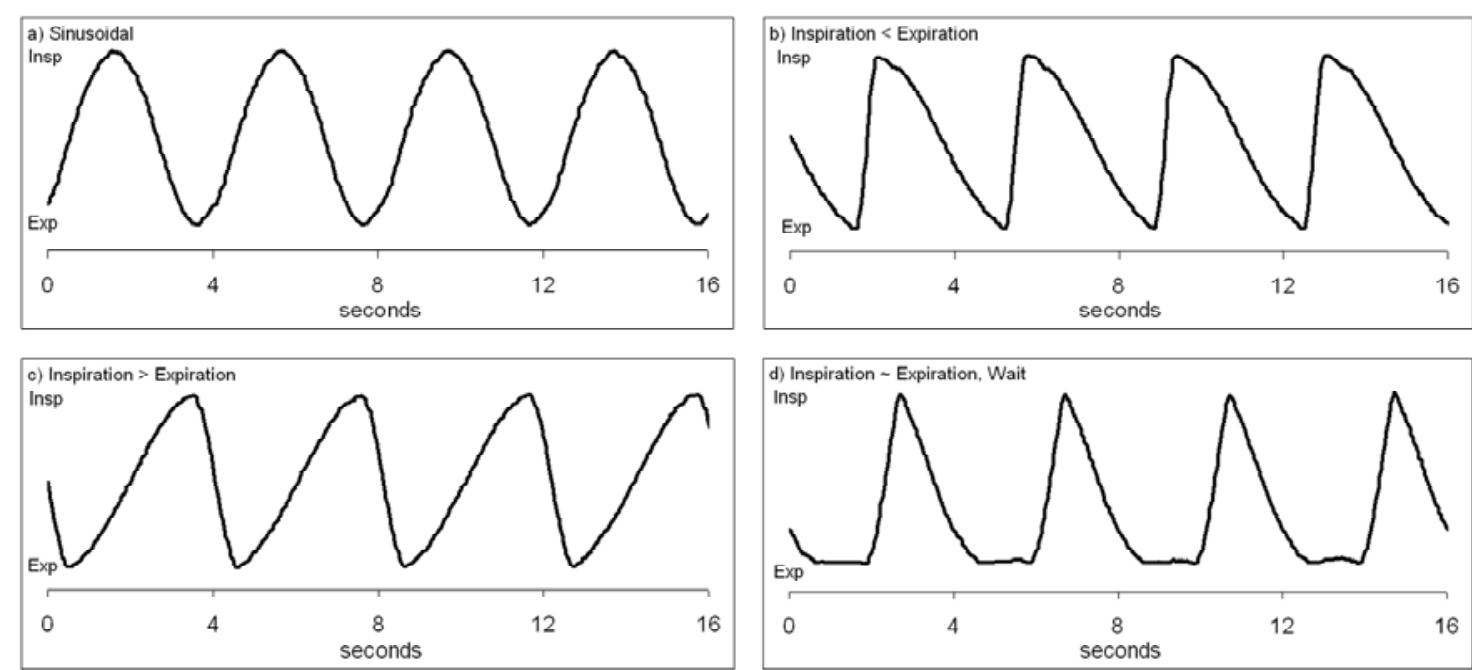

FIG. 2. Computer-generated breathing patterns used to move the small, medium, and large lesions: a) sinusoidal, b) inspiration less than expiration, c) inspiration greater than expiration, and d) equal inspiration and expiration followed by a rest period. The labels 'Insp' and 'Exp' correspond to the inferior and superior positions of the tumour, respectively, as they would in a patient. 


\section{II.B. Phantom imaging}

\section{II.B.1 $4 D C T$}

A Philips ${ }^{\circledR}$ Brilliance CT Big Bore scanner (Philips Medical Systems, Bothell, WA) with system version 2.3.0.17167 was used to acquire all CT images of the phantom. For 4DCT image reconstruction CT slices were binned into 10 phases (0$10 \%, 10-20 \%$, etc), with $0 \%$ relating to peak inspiration and $50 \%$ relating to peak expiration.

Reference 3DCT images were acquired of the stationary phantom housing the small, medium, and large lesions. Image acquisition settings for 3DCTs included 140 $\mathrm{kVp}$ tube voltage, $56 \mathrm{~mA}$ tube current, $3 \mathrm{~mm}$ slice thickness, $3 \mathrm{~mm}$ increment, $0.75 \mathrm{~s}$ rotation time, $500 \mathrm{~mm}$ field of view, 16x0.75 mm collimator, and couch pitch factor 0.688. Four-dimensional CT images were acquired using each of the 4 computergenerated breathing patterns with every combination of lesion size and amplitude with imaging settings adjusted to involve $0.44 \mathrm{~s}$ rotation time, $16 \times 1.5 \mathrm{~mm}$ collimator, and couch pitch factor of 0.1. Four-dimensional CT scans were then taken using the 6 SBRT lung patient breathing patterns matched to the patient-specific lesion size, and tumour amplitude. The couch pitch factor was adjusted according to Philips ${ }^{\circledR}$ look-up table recommendations for each patient-specific image based on the average breaths per minute of the moving phantom as calculated by the motion tracking system. Motion tracking for image reconstruction during 4DCT was done using the Bellows System (Philips Medical Systems, Bothell, WA). The phantom was scanned using the same imaging protocol as SBRT lung patients are scanned in clinic.

\section{II.B.2 CBCT}

Cone-beam CTs were acquired using a $\operatorname{Varian}^{\circledR}$ Trilogy linear accelerator fitted with an On-Board Imager ${ }^{\circledR}(\mathrm{OBI})$ with system version 1.4.13.0 (Varian Medical Systems, Palo Alto, CA). An imaging protocol 'Low Dose Thorax Cu' was commissioned in-house to acquire a CBCT with $110 \mathrm{kVp}$ tube voltage, $32 \mathrm{~mA}$ tube current, using a half-fan bow-tie filter fitted with an additional $0.15 \mathrm{~mm}$ sheet of copper. Each CBCT image was acquired over a full $360^{\circ}$ with the gantry rotating at $6 \% \mathrm{~s}$ and $2.5 \mathrm{~mm}$ slice thickness. Each CBCT projection was acquired roughly every $0.57^{\circ}$ with an x-ray pulse length of $20 \mathrm{~ms}$ resulting in about 650 projections per CBCT scan. The CBCT projections were reconstructed with a Ram-Lak convolution and a reconstruction matrix size of $512 \times 512$. 
Reference CBCT images were acquired of the stationary phantom housing the small, medium, and large lesions. Images were then acquired using each of the 4 computer-generated breathing patterns with every combination of lesion size and amplitude. Cone-beam CT scans were then taken using the 6 SBRT lung patient breathing patterns matched to the patient-specific lesion size, and tumour amplitude.

\section{II.C. Contouring ITVs}

Contouring of phantom ITVs in CBCT and 4DCT MIP images acquired of the lesions was done using the Auto Threshold tool in Focal software version 4.62.00 (Computerised Medical Systems, Inc., USA). Stationary and moving lesions in 4DCT MIP images in Focal were contoured using a window/level setting of 1700/500, while stationary lesions in CBCT images were contoured using a window/level setting of 1700/450. Different window values were required to contour moving lesions in CBCT as the contrast degraded with increasing amplitude. To standardise the window/level settings used in Auto Threshold contouring of moving lesions in CBCT the window value was adjusted for each amplitude until the anterior-posterior length of the lesion matched that of the stationary lesion in CBCT, while keeping a level value of 450 .

\section{II.D. Alignment of CBCT ITVs to 4DCT MIP ITV contours}

Aligning CBCT images with their corresponding reference 4DCT images was conducted in Focal. The CBCT images were registered to the 4DCT DICOM origin, accounting for any shifts of phantom alignment between 4DCT and CBCT. Both CBCT and 4DCT MIP images had an artificial sphere of enhanced CT values burnt into the DICOM origin for ease of visual alignment in Focal. The two artificial spheres were matched using window/level settings of 1100/-200 (4DCT) and 1100/300 (CBCT), and the initial transformation matrix values were noted. The CBCT lesion was then aligned to the 4DCT MIP ITV contour for Traces A, B, and C by visually matching the high-contrast central region to the centre of the 4DCT MIP ITV ensuring that the MIP ITV contour fully encompassed the CBCT ITV. Any changes in transformation matrix values were noted. For Trace D volumes it was difficult to be objective in a centre-to-centre alignment due to the CT contrast being weighted to one extreme and the sharper superior edge of the CBCT ITV being an attractive feature to use for alignment (Fig. 3). The centre-to-centre alignments of Trace D CBCT ITVs were therefore calculated by comparing the visible ITV centre to that of the 4DCT 
MIP ITV contour in the central coronal plane of the lesion. A subjective visual alignment was still carried out for lesions moved with Trace D; however, this involved using the sharper superior edge of the visible CBCT ITV in an 'edge-toedge' alignment.

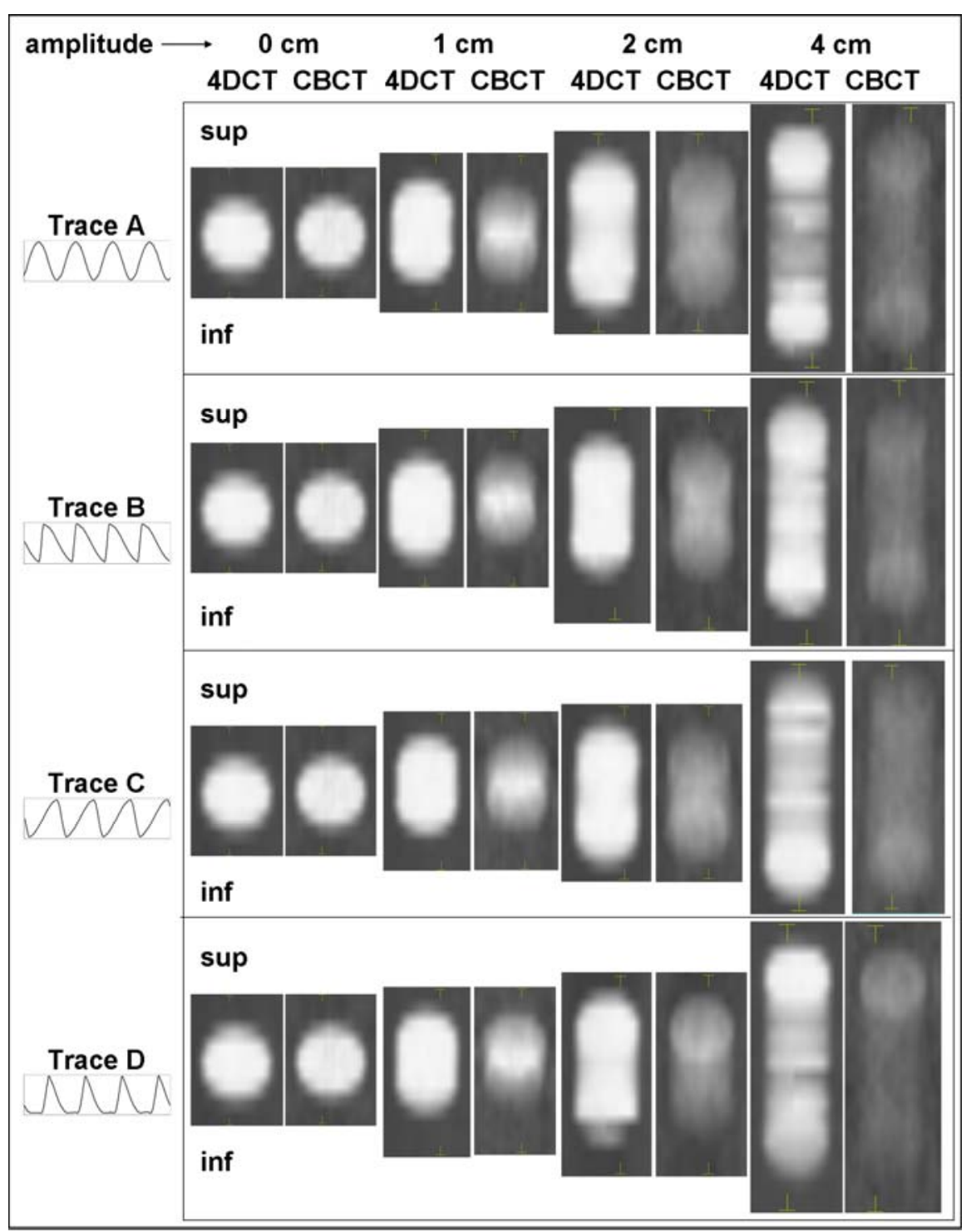

FIG. 3. The qualitative effect of amplitude on 4DCT MIP and CBCT ITVs for the small lesion moving with computer-generated breathing patterns Trace A, B, C, and D. 


\section{RESULTS}

\section{III.A Computer-generated breathing patterns}

\section{III.A.1 Qualitative effect of motion on ITVS}

Up to a $2 \mathrm{~cm}$ peak to peak amplitude the 4DCT MIP ITVs captured the motion of the lesions with a roughly even distribution of contrast (Fig. 3, small lesion example). Banding artefacts became evident when the amplitude was set to $4 \mathrm{~cm}$, and in the $2 \mathrm{~cm}$ Trace D 4DCT MIP where the lesion spent less time at the inferior extreme and was not accurately captured. Cone-beam CT ITVs were more difficult to visualise, particularly at the extremes, than the 4DCT MIP ITVs as motion degraded the contrast between the Perspex and wood materials. For Trace A, B, and C the extremes of the CBCT ITVs were of equal visual intensity and the highest contrast region roughly correlated to the centre of motion. For Trace D however, contrast was weighted to the superior extreme, and the centre of the visible CBCT volume no longer correlated to the centre of motion.

\section{III.A.2 ITV and ITV length}

The measured 4DCT MIP ITVs and ITV lengths were compared to the true ITVs and ITV lengths for the small, medium, and large lesions moved $0 \mathrm{~cm}, 1 \mathrm{~cm}, 2$ $\mathrm{cm}$, and $4 \mathrm{~cm}$ with the 4 computer-generated breathing patterns (Fig. 4). True ITV and ITV lengths were calculated based on an object of known geometry moved a known distance. 4DCT MIP ITV values of moving targets were smaller than the true volumes for all lesion sizes irrespective of breathing trace and amplitude, with a distinct trend in volume reduction from true volume with increasing amplitude. Volumes were reduced by up to $20 \%$ and shortened by up to $7 \mathrm{~mm}$ with motion. Trace A (sinusoidal motion) typically showed the smallest reductions in 4DCT MIP ITVs and ITV lengths when motion was introduced, particularly for the medium and large lesions.

The measured CBCT ITVs and ITV lengths were compared to the true ITVs and ITV lengths for the small, medium, and large lesions moved $0 \mathrm{~cm}, 1 \mathrm{~cm}, 2 \mathrm{~cm}$, and $4 \mathrm{~cm}$ with the 4 computer-generated breathing patterns (Fig. 5). The CBCT ITVs were smaller and shorter than the true ITVs and ITV lengths for all lesion sizes irrespective of breathing trace and amplitude. Cone-beam volumes fared worse than 4DCT volumes being reduced by up to $30 \%$ and shortened by up to $11 \mathrm{~mm}$ with motion. There was not a distinct trend in reduction of CBCT ITV with increasing amplitude as was evident in the 4DCT data; however, this is related to the various 
window/level settings used to auto-threshold contour CBCT ITVs of moving objects when only one window/level setting was required to contour 4DCT MIP ITVs. Again, Trace A (sinusoidal motion) typically showed the smallest reductions in measured CBCT ITVs and ITV lengths when motion was introduced.
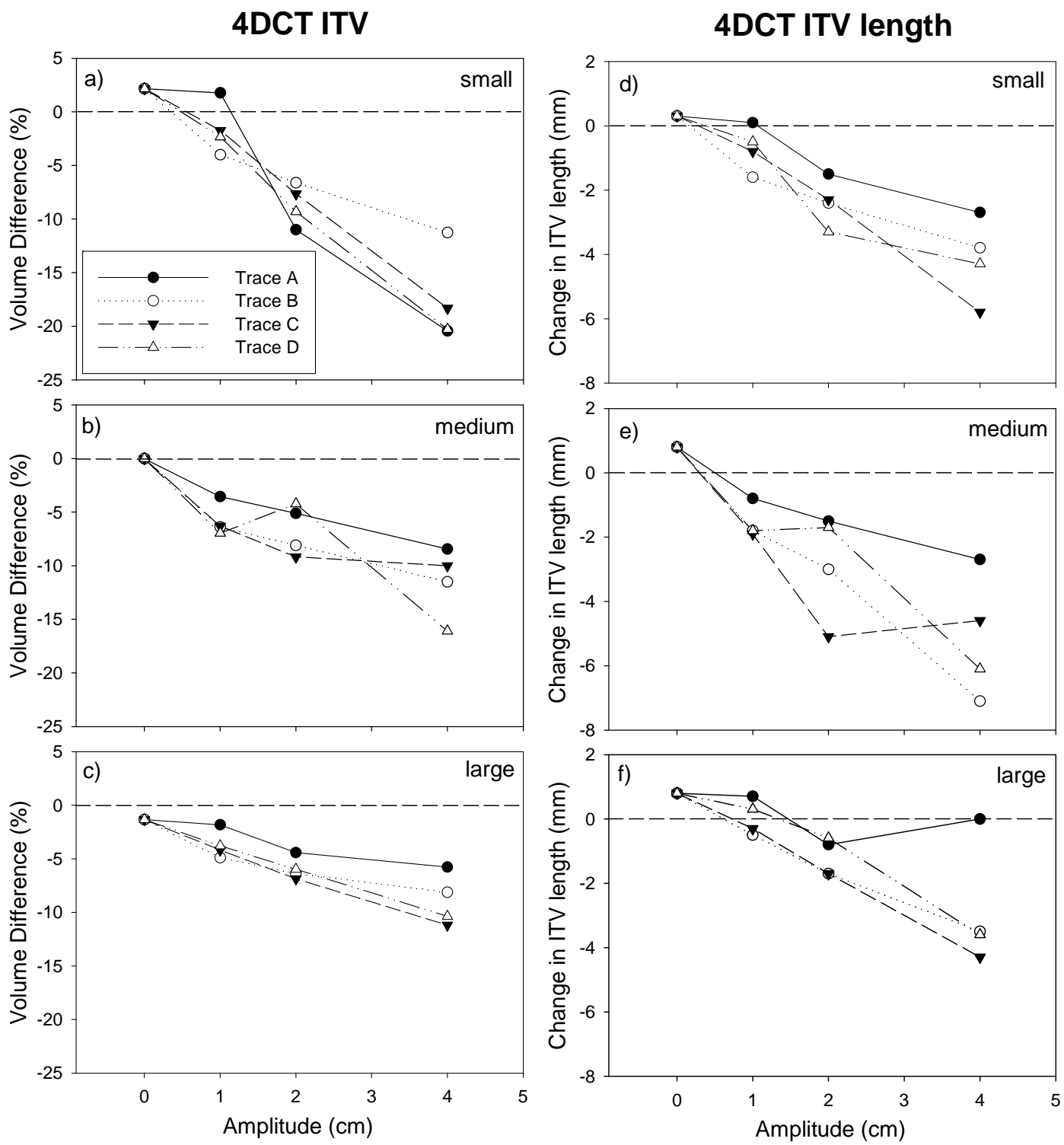

FIG. 4. Measured 4DCT MIP ITV and ITV lengths compared to true 4DCT MIP ITV and ITV lengths for small, medium, and large lesions moved $0 \mathrm{~cm}, 1 \mathrm{~cm}, 2 \mathrm{~cm}$, and $4 \mathrm{~cm}$ with computer-generated breathing patterns Trace A, B, C, and D. Plots a), b), and c) illustrate the relative volume change in ITV (\%), and plots d), e), and f) illustrate the change in ITV length (mm). The dotted horizontal lines at $0 \%$ and $0 \mathrm{~mm}$ represent no change from the true ITVs and true ITV lengths, respectively (i.e. a lesion of known geometry was moved a known distance). 
The patterns of ITV length shortening followed the same patterns of ITV reduction for both imaging modalities indicating that volume was being underrepresented at the extremes of motion.
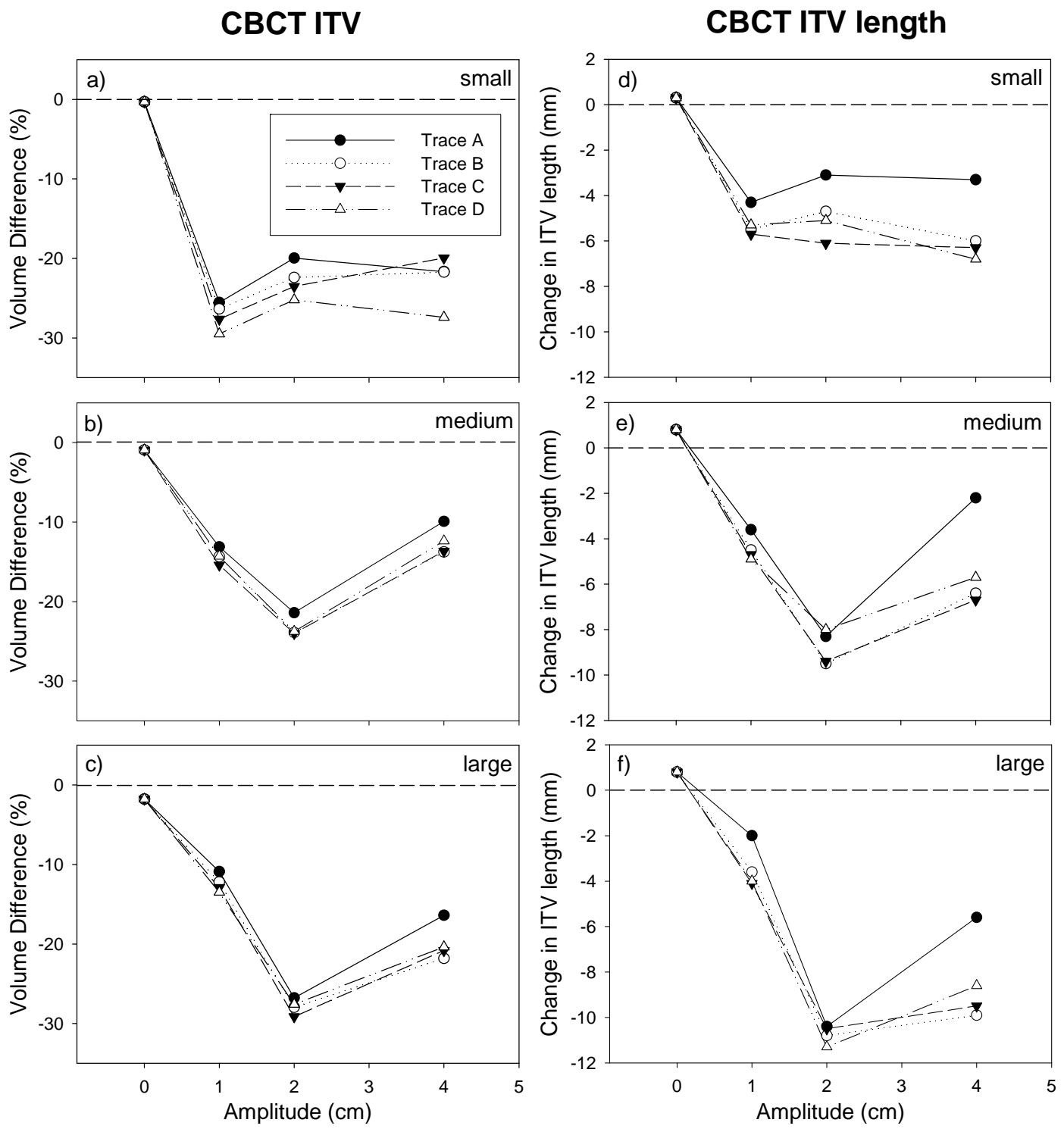

FIG. 5. Measured CBCT ITV and ITV lengths compared to true CBCT ITV and ITV lengths for small, medium, and large lesions moved $0 \mathrm{~cm}, 1 \mathrm{~cm}, 2 \mathrm{~cm}$, and $4 \mathrm{~cm}$ with computer-generated breathing patterns Trace A, B, C, and D. Plots a), b), and c) illustrate the relative volume change in ITV (\%), and plots d), e), and f) illustrate the change in ITV length (mm). The dotted horizontal lines at $0 \%$ and 0 $\mathrm{mm}$ represent no change from the true ITVs and true ITV lengths, respectively (i.e. a lesion of known geometry was moved a known distance). 


\section{III.A.3 Alignment of ITVs}

The alignments of CBCT ITVs to 4DCT MIP ITV contours were generally limited to image sets from the same breathing pattern between 4DCT and CBCT. With motion limited along the SI axis the SI shifts were of particular interest for analysing the translational shifts required. The SI shifts required to visually align CBCT ITVs to 4DCT MIP ITV contours of lesions moved with Trace A, B, and C are listed in Table I, as are the results from both centre-to-centre alignments and edge-toedge alignments for lesions moved with Trace D.

TABLE I. SI shifts required to visually align small, medium, and large lesions moved $1 \mathrm{~cm}, 2$ $\mathrm{cm}$, and $4 \mathrm{~cm}$ with computer-generated patterns Trace A, B, C, and D. Two techniques were used to align volumes from Trace D; centre-to-centre, and edge-to-edge.

\begin{tabular}{ccccccc}
\hline \hline \multirow{2}{*}{ Insert size } & $\begin{array}{c}\text { Amplitude } \\
(\mathrm{cm})\end{array}$ & Trace A & Trace B & Trace C & $\begin{array}{c}\text { Trace D } \\
\text { cntr-to-cntr }\end{array}$ & $\begin{array}{c}\text { Trace D } \\
\text { edge-to-edge }\end{array}$ \\
\cline { 3 - 7 } small & 1 & -0.9 & 0.9 & 0.0 & -1.5 & -1.2 \\
& 2 & -0.6 & -1.2 & 0.0 & -1.2 & 0.0 \\
\multirow{3}{*}{ medium } & 4 & -0.4 & 0.7 & 0.1 & -1.2 & 0.2 \\
& 1 & 0.0 & -1.5 & -1.1 & 0.6 & -1.0 \\
& 2 & 0.0 & -0.8 & 0.0 & -0.9 & -0.4 \\
large & 4 & -0.8 & -2.2 & -1.1 & -2.3 & -1.5 \\
& 1 & 0.1 & 2.1 & 0.3 & -2.5 & -1.1 \\
& 2 & -0.5 & -0.8 & 0.0 & -1.1 & -0.2 \\
\hline \hline
\end{tabular}

The maximum SI shifts required for Trace A, B, C, and D (centre-to-centre) were 0.9 $\mathrm{mm}, 2.2 \mathrm{~mm}, 1.1 \mathrm{~mm}$, and $2.5 \mathrm{~mm}$, respectively. The lowest maximum SI shifts were measured for sinusoidal motion while the largest SI shifts were required for Trace D when a centre-to-centre alignment technique was used. Comparing the two Trace D columns the edge-to-edge alignments resulted in a better agreement in SI shifts than the centre-to-centre alignments. There were no trends with lesion size or amplitude. To determine the magnitude of shift of the centre of the visible CBCT ITV from Trace D away from the centre of motion, centrepoints of CBCT ITVs from Trace A (sinusoidal) and Trace D were calculated and compared to centrepoints of 4DCT ITVs from Trace A; where a sinusoidal pattern would cause the least shift of the CBCT ITV centre away from the centre of motion (Table II). 
TABLE II. SI shifts calculated to match CBCT ITV centres to 4DCT MIP ITV centres of the small, medium, and large lesions moved $1 \mathrm{~cm}, 2 \mathrm{~cm}$, and $4 \mathrm{~cm}$, with sinusoidal patterns and Trace D.

\begin{tabular}{|c|c|c|c|c|c|}
\hline $\begin{array}{l}\text { Lesion } \\
\text { Size }\end{array}$ & $\begin{array}{l}\text { Amplitude } \\
\text { (cm) }\end{array}$ & $\begin{array}{l}\text { CBCT Sine } \\
\text { vs } \\
\text { 4DCT Sine } \\
\text { (mm) }\end{array}$ & $\begin{array}{c}\text { CBCT Trace D } \\
\text { vs } \\
\text { 4DCT Trace D } \\
(\mathrm{mm})\end{array}$ & $\begin{array}{c}\text { CBCT Trace D } \\
\text { vs } \\
\text { 4DCT Sine } \\
(\mathrm{mm})\end{array}$ & $\begin{array}{c}\text { CBCT Trace D } \\
\text { vs } \\
\text { CBCT Sine } \\
(\mathrm{mm})\end{array}$ \\
\hline \multirow[t]{3}{*}{ small } & 1 & -0.2 & -1.5 & -1.5 & -1.3 \\
\hline & 2 & 1.2 & -1.2 & 0.3 & -0.9 \\
\hline & 4 & -0.3 & -1.2 & -2.9 & -2.6 \\
\hline \multirow[t]{3}{*}{ medium } & 1 & -1.3 & 0.6 & -2.6 & -1.3 \\
\hline & 2 & 0.3 & -0.9 & -2.3 & -2.7 \\
\hline & 4 & 0.2 & -2.3 & -1.0 & -1.2 \\
\hline \multirow[t]{3}{*}{ large } & 1 & -0.0 & -2.5 & -2.5 & -2.4 \\
\hline & 2 & 0.1 & -1.1 & -2.4 & -2.5 \\
\hline & 4 & -0.1 & -2.1 & -3.7 & -3.6 \\
\hline
\end{tabular}

Comparing the values in the 'Sine vs Sine' column to those in the 'Trace D vs Trace D' column it can be seen that larger shifts are more often required to align the centrepoints of CBCT and 4DCT MIP ITVs from Trace D than from sinusoidal motion. The 'Trace D vs 4DCT Sine' column shows that in all cases but one the centrepoints of Trace D CBCT ITVs were superior (negative shift) to the sinusoidal 4DCT MIP ITV centres with required shifts up to $3.7 \mathrm{~mm}$. The 'Trace D vs CBCT Sine' column lists the shifts required to match the CBCT ITVs from Trace D to those from sinusoidal motion and given that they are all negative this shows that Trace D causes a superior shift in the centrepoint of the CBCT ITV away from the centre of motion.

\section{III.B SBRT lung patient breathing patterns}

\section{III.B.1 Qualitative effect of motion on ITVs}

Since the maximum amplitudes for the patients' lesions were all less than $1 \mathrm{~cm}$ there were no banding artefacts in the phantom 4DCT MIP ITVs, and most of the lesions on CBCT had similar contrast to their corresponding 4DCT MIP images. The exception was the CBCT of Patient 6 that had been notably blurred as a result of being moved with the largest amplitude of approximately $1 \mathrm{~cm}$.

\section{III.B.2 ITV, ITV length and alignment}

Table III lists the comparison of measured ITVs to true ITVs for 4DCT MIP and CBCT images of the phantom moved with the 6 SBRT lung patient breathing 
patterns. The CBCT volumes are also compared to 4DCT volumes because in clinical patient images there is no 'true ITV' so 4DCT MIP ITVs become the gold standard. This will allow comparison to data presented in the literature.

TABLE III. Measured 4DCT MIP and CBCT ITVs of lesions moved with the 6 SBRT lung patient breathing traces compared to true ITVs and relative to each other. Patient traces are identified by insert size, amplitude, and true ITV. Required SI shifts to visually align lesions moved with SBRT patient traces are listed in the rightmost column.

\begin{tabular}{|c|c|c|c|c|c|c|c|c|c|}
\hline \multirow{2}{*}{$\begin{array}{c}\text { Patient } \\
\text { Trace }\end{array}$} & \multirow{2}{*}{$\begin{array}{l}\text { Insert } \\
\text { size }\end{array}$} & \multirow{2}{*}{$\begin{array}{l}\text { Amp } \\
(\mathrm{mm})\end{array}$} & \multirow{2}{*}{$\begin{array}{c}\text { True } \\
\text { ITV (cc) }\end{array}$} & \multicolumn{2}{|c|}{$4 \mathrm{DCT}$} & \multicolumn{2}{|c|}{ CBCT } & \multirow{2}{*}{$\begin{array}{c}\text { CBCT vs } \\
\text { 4DCT } \\
(\%)\end{array}$} & \multirow{2}{*}{$\begin{array}{c}\text { Shift } \\
\text { SI (mm) }\end{array}$} \\
\hline & & & & $\begin{array}{c}\text { Meas. } \\
\text { ITV (cc) }\end{array}$ & $\begin{array}{l}\text { Diff } \\
(\%)\end{array}$ & $\begin{array}{c}\text { Meas. } \\
\text { ITV (cc) }\end{array}$ & $\begin{array}{l}\text { Diff } \\
(\%)\end{array}$ & & \\
\hline 1 & medium & 4.5 & 25.26 & 22.69 & -10 & 23.17 & -8 & 2 & -0.6 \\
\hline 2 & small & 3.5 & 3.44 & 3.09 & -10 & 3.01 & -13 & -3 & -0.6 \\
\hline 3 & medium & 4.5 & 25.26 & 23.93 & -5 & 23.00 & -9 & -4 & 0.6 \\
\hline 4 & small & 6.5 & 4.11 & 3.33 & -19 & 3.06 & -26 & -8 & -0.4 \\
\hline 5 & small & 6.0 & 4.00 & 3.38 & -15 & 3.09 & -23 & -9 & 0.0 \\
\hline 6 & small & 9.0 & 4.68 & 4.14 & -11 & 3.23 & -31 & -22 & 1.1 \\
\hline
\end{tabular}

The 4DCT MIP and CBCT ITVs were always smaller than the true ITVs. For 4DCT MIPs the lesion ITVs were reduced by up to $20 \%$ while CBCT ITVs were reduced by up to $30 \%$. Furthermore, in all cases but one the CBCT ITV was less than the 4DCT MIP ITV. For the small lesion CBCT ITVs showed a distinctly decreasing trend with amplitude though this trend was not evident for the medium lesion since the two motions had the same maximum amplitudes. The reductions of 4DCT MIP ITVs were far worse for patient breathing patterns (variable amplitudes) than for constant amplitude breathing patterns (Traces A-D). The small lesion moved with variable amplitudes up to $1 \mathrm{~cm}$ had 4DCT MIP ITV reductions of up to $20 \%$, while a constant amplitude of $1 \mathrm{~cm}$ resulted in much lower ITV reductions of up to 5\% (Fig. 4). Conebeam CT ITV reductions remained on the same magnitude for both variable amplitudes and constant amplitudes (Table III and Fig. 5).

The measured 4DCT MIP and CBCT ITV lengths compared to the true ITV lengths for the small and medium lesions moved with the 6 SBRT lung patient breathing patterns is presented in Figure 6. In all cases the lengths of 4DCT MIP ITVs and CBCT ITVs were less than the true ITV lengths. Furthermore, the CBCT ITV was consistently shorter than the 4DCT MIP ITV in all cases for the small \& medium lesions. There was a tendency of 4DCT MIP ITV lengths to shorten with increasing amplitude, though a more distinct trend in shortening of CBCT ITV lengths with 
increasing amplitude was evident. Volumes at the extremes of motion are not being accurately reconstructed by either 4DCT MIP or CBCT when the extremes of motion re not always reached, with CBCT being the least accurate in this respect.

For alignment of CBCT ITV to the 4DCT MIP ITV contour from patient breathing traces the greatest SI shift was $1.1 \mathrm{~mm}$ (Table III) and this related to the CBCT ITV length that was shortened by the largest amount of $6.6 \mathrm{~mm}$. Large misalignments were not seen for patient breathing patterns due to the maximum amplitudes all being less than $1 \mathrm{~cm}$.

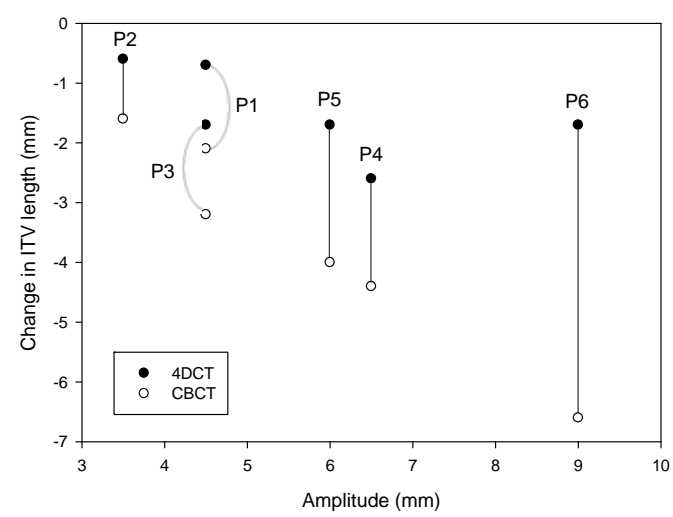

FIG. 6. Change in 4DCT MIP and CBCT ITV length as a function of amplitude for the small and medium lesions moved with SBRT lung patient breathing patterns. The $0 \mathrm{~mm}$ line represents the true ITV lengths and measurement points are identified and connected by the patient breathing trace used.

\section{DISCUSSION}

By imaging various moving phantom configurations it was found that large and varied amplitudes of motion could reduce the 4DCT ITVs by up to $20 \%$ and the CBCT ITVs by up to $30 \%$, with CBCT imaging less capable of reproducing the ITVs for moving lesions. Sinusoidal breathing patterns resulted in the closest agreements in the majority of parameters measured. This finding highlights the importance of quantifying the effect that more clinically realistic breathing patterns have on the ability of 4DCT MIP and CBCT imaging to accurately capture a moving target. Four computer-generated and 6 patient-specific patterns of motion were studied and it was lesions that exhibited a rest period following expiration that revealed two interesting findings. Firstly, the superior edge of the CBCT ITV was more clearly visible 
compared to the inferior edge that was blurred and, in some cases, indistinguishable from the background. Secondly, there was a shift of the centre of the visible CBCT ITV away from the centre of motion, particularly for smaller lesions moving with large peak to peak amplitudes. If the centre of a CBCT ITV with one blurred or missing edge was matched to the centre of the 4DCT MIP ITV, then this could result in a misalignment of SBRT lung patients. Given the complexity that some patient breathing patterns can exhibit, including large amplitude variations and sizeable baseline drifts, the findings of this study are limited to breathing patterns with rest periods similar to those investigated in this study.

The advent of 4D CBCT has recently become clinically available with the ability to acquire a set of 3D CBCT images that illustrate the various tumour positions during image acquisition. ${ }^{6,7}$ By increasing the scan time and acquiring more projections in all breath phases, this imaging modality has the ability to almost eliminate the blurred images that conventional 3D CBCT reconstructs of a moving target, enabling a more accurate patient alignment if the minimum and maximum excursions are clearly visible. ${ }^{5,8}$ The downside at this point in time is $4 \mathrm{D}$ CBCT is not as commonly available as 3D CBCT. In the absence of $4 \mathrm{D} \mathrm{CBCT,} \mathrm{the} \mathrm{below}$ discussion outlines the qualitative and quantitative assessment of the measured volumes, the scope of the analysis, and the clinical recommendations based on the measured results.

\section{IV.A Qualitative analysis}

Banding and blurring artefacts in 4DCT MIP images from sinusoidal and irregular motion have been reported in the literature, ${ }^{9-11}$ and were particularly evident here for the small lesion moving greater than $2 \mathrm{~cm}$. It may be worth considering reducing the couch pitch factor and/or reducing the slice thickness for when the imaging plane is directly overtop of and capturing lesions with large displacements or fast motion.

Imaging moving objects with CBCT resulted in a marked reduction in contrast compared to that of 4DCT MIP imaging. CBCT image reconstruction makes use of every pixel in each projection that results in an averaging of the CT numbers across the image set, giving a lower contrast between Perspex and lung that worsened as amplitude increased. The visual effect on CBCT ITVs from patterns similar to Trace 
D presented here, involving the superior edge of the CBCT ITV being more welldefined than the inferior edge, agrees with that reported in the literature. ${ }^{5}$

\section{IV.B Quantitative analysis: ITV and ITV length}

When motion was introduced both 4DCT MIP and CBCT ITVs experienced reductions in volume and length with $\mathrm{CBCT}$ faring the worst in the majority of cases. The inability of both imaging modalities to accurately reconstruct tumour volumes is linked to their respective imaging reconstruction processes.

For 4DCT image acquisition the couch pitch factor is calculated based on the average breath length and does not take amplitude of tumour motion into consideration. A tumour moving with an amplitude of $1 \mathrm{~cm}$ is moving much slower and is therefore more easily captured by 4DCT than one moving $4 \mathrm{~cm}$ if the breath lengths are identical. As referenced above, large displacements in 4DCT results in missing data and imaging artefacts. If a lesion moving irregularly travels to its maximum amplitude when outside the 4DCT imaging plane then this position will not be visible in the image reconstruction. The larger the maximum amplitude moved, the larger the potential error (Fig. 6). This may be happening in patient imaging but without knowing the maximum amplitude reached by the tumour the true errors in 4DCT ITVs may be underrepresented.

Every CBCT projection is used in image reconstruction and results in a CT number averaging effect. If the lesion only travels to a large amplitude once then the number of CBCT projections capturing the lesion at that amplitude will be largely outweighed and averaged out by the number of projections of the lesion not at that position.

The literature includes a variety of phantom studies that have quantified 4DCT MIP ITVs of objects moving sinusoidally ${ }^{2,3,10}$ and irregularly ${ }^{1,10}$ and report similar results as those presented here. Various studies have also investigated the effect of sinusoidal patterns on CBCT ITVs ${ }^{2,4,5}$, while only one has looked at irregular breathing patterns ${ }^{5}$. Wang (2007) found a lesser reduction in CBCT ITV of $10 \%$ where close to $30 \%$ was reported here and is potentially due to them comparing CBCT ITVs to 4DCT ITVs (not true ITV), or their use of a high-contrast steel 'lesion' which is less clinically realistic than the imaging contrast of Perspex in wood. Though Vergalasova (2011) did not quantify the volumetric effect on CBCT ITV from irregular breathing patterns they did assess the changes in internal target area, and 
showed a greater reduction in area for smaller lesions, larger amplitudes, and smaller inspiration to expiration ratios. Where Vergalasova (2011) stated that there was a potential for CBCT ITV underestimation, the results presented here showed that there is an underestimation of CBCT ITV for any amount of motion introduced regardless of lesion size, motion amplitude, breathing pattern, and slight variations in window levels for contouring. This project is the first to quantify the volumetric effect of irregular breathing patterns on CBCT ITVs.

Various authors have also quantified the effect on 4DCT MIP ITV lengths for sinusoidal motion ${ }^{10}$ as well as for irregular motion, ${ }^{1,10}$ however, it is difficult to compare studies as a variety of imaging slice thicknesses and lesion shapes were used. Though the margins used to expand the 4DCT MIP ITV to the PTV are typically greater than $5 \mathrm{~mm},{ }^{12}$ it was seen from this and other studies that the 4DCT MIP ITV length can be shorter by $7 \mathrm{~mm}$ for large motion and irregular breathing patterns. This leaves little room for error in alignment if there is only $10 \mathrm{~mm}$ in which to move superiorly and inferiorly. Cases such as these may result in an underdosage of the PTV. ${ }^{13}$ The finding that 4DCT MIP ITV lengths were shortened for lesions that moved with variable amplitudes is clinically relevant as patients breathe with different degrees of inspiration and expiration, and this would move their tumour to a variety of amplitudes. Though clinically relevant, this aspect is not well studied in the literature.

\section{IV.C Quantitative analysis: ITV alignment}

Even though the volumes and lengths of CBCT ITVs were reduced by large amounts, the alignments of CBCT ITVs to 4DCT MIP ITVs from Trace A, B, and C, as well as all patient breathing patterns approximated on the phantom were within 2 $\mathrm{mm}$. It is therefore appropriate to use centre-to-centre matching of CBCT ITVs to 4DCT MIP ITVs without chance of gross misalignment for these patterns and for patient breathing patterns similar to those used here that moved the lesion less than 1 $\mathrm{cm}$. For Trace D it was found that there was a shift in CBCT ITV centres in the superior direction as a result of the inferior extreme of motion not being accurately captured. A subjective edge-to-edge alignment was a more appropriate method of alignment than a centre-to-centre alignment method for this pattern of motion.

Various authors have conducted similar alignments between CBCT and 4DCT images for phantoms moving with sinusoidal motion, ${ }^{2,14}$ though this study is the first to report on the error in alignment from non-sinusoidal breathing patterns. The results 
reported in Wang (2007), Jiang (2012), and here showed that alignments of CBCT ITVs with 4DCT ITVs for sinusoidal breathing patterns around 4 seconds in length and amplitudes less than $4 \mathrm{~cm}$ would not result in gross misalignments of a patient. Vergalasova (2011) did not align their CBCTs to the corresponding 4DCTs though they state that with increasing time spent in expiration there would be a greater likelihood of localisation error. The results presented here proved that there was a greater localisation error for patterns of motion with a rest period than for regular sinusoidal motion.

\section{IV.D Limitations of study}

This project limited itself to 4 second breath cycle lengths, compared the same breathing pattern between $4 \mathrm{DCT}$ and $\mathrm{CBCT}$, and phantom motion was constrained to the SI axis. Although patients have been observed to breathe with the same pattern between 4DCT and CBCT imaging, and lung tumours generally move more in the SI direction, ${ }^{15}$ further investigations involving 3D tumour motion and different breathing patterns between 4DCT and CBCT would be worthwhile. This project used the same 4DCT and CBCT image acquisition settings that our clinical patient images would have been acquired with including slice thickness, rotation speed, pitch of the acquisition, helical for 4DCT, and half-fan beam for CBCT. The findings in this project are limited to Philips ${ }^{\circledR}$ and $\operatorname{Varian}^{\circledR}$ equipment and to images acquired using the same settings as indicated.

\section{IV.E Clinical implications and recommendations}

The clinical implications of errors in ITV from motion have resulted in two recommendations to change clinical practice; one based on the findings from 4DCT MIP ITVs, the other on the CBCT ITV findings.

Having a 4DCT MIP ITV that is smaller and shorter than the true ITV would result in an error in 4DCT MIP ITV contouring for patient treatment planning that would be carried right through to patient treatment. It is therefore recommended that the creation of 4DCT MIP ITV to PTV margins for SBRT lung lesions should account for any loss of volume at the extremes of motion as a result of peak to peak amplitudes of motion greater then $2 \mathrm{~cm}$. Since the volume that is ultimately treated is built on the 4DCT MIP ITV contour an erroneous ITV contour from the beginning would introduce a systematic error that would propagate through to the patient's 
treatment. Typical ITV to PTV margins involve an additional $5 \mathrm{~mm}$ in the axial planes, and a full $10 \mathrm{~mm}$ in the SI directions ${ }^{12}$ to account for setup errors as well as larger tumour motion on treatment day than that imaged during 4DCT scanning. It is not until the lesions move with amplitudes larger than $2 \mathrm{~cm}$ that ITV to PTV margins would warrant consideration for anisotropic margins in the direction of greatest motion.

The clinical impact of a CBCT ITV that is smaller and shorter than the true ITV is not as serious as that of a smaller 4DCT MIP ITV provided that the centre of the visible CBCT ITV correlates to the centre of lesion motion. However, if irregular breathing patterns such as Trace D reduced the visibility of the inferior CBCT ITV edge and shifted the centre of the visible CBCT ITV away from the centre of motion then a centre-to-centre match would cause a misalignment between the CBCT and the 4DCT MIP ITV contour (Fig. 1). Based on the findings for CBCT ITVs it is recommended that the patient's breathing pattern becomes a tool to align the stereotactic lung patient. If their breathing pattern exhibits a rest period following expiration and no sizeable baseline drift then the superior edge of their lesion should be better defined than the inferior edge and can be used for edge-to-edge alignment of the CBCT ITV to the 4DCT MIP ITV contour as opposed to centre-to-centre matching that is currently being done. For patients not exhibiting this type of breathing trace it was shown that a centre-to-centre matching of the CBCT ITV to the 4DCT MIP ITV contour was an appropriate method of alignment.

\section{CONCLUSION}

The effectiveness of stereotactic lung radiotherapy is dependent on the accuracy of the imaging used for treatment planning and patient alignment. As the majority of patients breathe with irregularities in pattern and amplitude the investigation of more clinically realistic motion patterns has been an important one. Isotropic $5 \mathrm{~mm}$ 4DCT MIP ITV to PTV margins would be suitable for lesions that move with amplitudes less than $2 \mathrm{~cm}$ as 4DCT MIP ITV reductions are not large. Anisotropic margins in the direction of motion should be considered for lesions moving greater than $2 \mathrm{~cm}$. Breathing patterns should be acquired during CBCT imaging of the stereotactic lung patient and if the pattern has a rest period following expiration then an edge-to-edge alignment technique should be adopted. This project has highlighted a need to monitor tumour amplitude and patient breathing patterns 
during both planning $\mathrm{CT}$ imaging and $\mathrm{CBCT}$ imaging as this additional information can be used to more accurately contour the 4DCT MIP ITV and align the lung patient prior to their stereotactic radiotherapy treatment.

\section{REFERENCES}

${ }^{1}$ C. E. Noel and P. J. Parikh, Med Phys 38 (5), 2430-2438 (2011).

${ }^{2}$ Z. Wang, Q. J. Wu, L. B. Marks, N. Larrier and F. F. Yin, Int J Radiat Oncol Biol Phys 69 (5), 1618-1624 (2007).

${ }^{3}$ R. W. Underberg, F. J. Lagerwaard, B. J. Slotman, J. P. Cuijpers and S. Senan, Int J Radiat Oncol Biol Phys 63 (1), 253-260 (2005).

${ }^{4}$ J. Y. Song, T. K. Nam, S. J. Ahn, W. K. Chung, M. S. Yoon and B. S. Nah, Med Dosim 34 (2), 117-125 (2009).

${ }^{5}$ I. Vergalasova, J. Maurer and F. F. Yin, Med Phys 38 (8), 4689-4699 (2011).

${ }^{6}$ T. Li, L. Xing, P. Munro, C. McGuinness, M. Chao, Y. Yang, B. Loo and A. Koong, Med Phys 33 (10), 3825-3833 (2006).

${ }^{7}$ J. Lu, T. M. Guerrero, P. Munro, A. Jeung, P. C. Chi, P. Balter, X. R. Zhu, R. Mohan and T. Pan, Med Phys 34 (9), 3520-3529 (2007).

${ }^{8}$ T. G. Purdie, D. J. Moseley, J. P. Bissonnette, M. B. Sharpe, K. Franks, A. Bezjak and D. A. Jaffray, Acta Oncol 45 (7), 915-922 (2006).

${ }^{9}$ P. Keall, G. Starkschall, H. Shukla, K. M. Forster, V. Ortiz, C. W. Stevens, S. S. Vedam, R. George, T. Guerrero and R. Mohan, Phys Med Biol 49 (10), 2053-2067 (2004).

${ }^{10}$ K. Park, L. Huang, H. Gagne and L. Papiez, Int J Radiat Oncol Biol Phys 73 (2), 618-625 (2009).

${ }^{11}$ W. T. Watkins, Med Phys 37 (6), 2855-2861 (2010).

${ }^{12}$ S. H. Benedict, K. M. Yenice, D. Followill, J. M. Galvin, W. Hinson, B. Kavanagh, P. Keall, M. Lovelock, S. Meeks, L. Papiez, T. Purdie, R. Sadagopan, M. C. Schell, B. Salter, D. J. Schlesinger, A. S. Shiu, T. Solberg, D. Y. Song, V. Stieber, R. Timmerman, W. A. Tome, D. Verellen, L. Wang and F. F. Yin, Med Phys 37 (8), 4078-4101 (2010).

${ }^{13}$ L. Huang, K. Park, T. Boike, P. Lee, L. Papiez, T. Solberg, C. Ding and R. D. Timmerman, Radiother Oncol 96 (1), 48-54 (2010).

${ }^{14}$ B. Jiang, J. Dai, Y. Zhang, K. Zhang, K. Men, Z. Zhou, J. Liang and L. Wang, Med Dosim 37 (1), 47-52 (2012).

${ }^{15}$ Y. Seppenwoolde, H. Shirato, K. Kitamura, S. Shimizu, M. van Herk, J. V. Lebesque and K. Miyasaka, Int J Radiat Oncol Biol Phys 53 (4), 822-834 (2002). 